\section{Rheumatology 4.0: big data, wearables and diagnosis by computer}

\begin{abstract}
We must dare to think 'unthinkable' thoughts. We must learn to explore all the options and possibilities that confront us in a complex and rapidly changing world. J. William Fulbright, US Politician. 9 April 1905-9 February 1995

Nothing is unthinkable, nothing impossible to the balanced person, provided it comes out of the needs of life and is dedicated to life's further development. Lewis Mumford, US historian, sociologist, philosopher and literary critic. 19 October 1895-26 January 1990
\end{abstract}

Let's jump into the future, about 10 years from now. Sandy Myers (fictional name) is a 44-year-old female IT specialist. She is suffering from joint pain, morning stiffness, swollen finger joints and toes, as well as fatigue since 4 months. What should she do now? You would say, she should see rheumatologist as soon as possible and attend an early arthritis clinic. But will we have enough rheumatologists in 10 years, will this be common practice? Instead, she walks into the living room in the morning and addresses Alessia (fictional name), her cloud-based voice service machine. She tells Alessia about her health problems, and the machine takes a detailed history asking her several questions, which have been elaborated by a EULAR initiative (EULAR recommendations on core rheumatology questions in cloud-based voice service. Annals of the Rheumatic Diseases 2025): how can I help you?, where do you have your pain?, is it more pronounced in the morning?, do non-steroidals help?, do you feel like having an influenza? and some others. The answers are entered online into a structured patient history databank.

Sandy is now asked by Alessia to perform a self-examination that is helped by a large screen on her living room wall. Showing examples of diseased joints and with the help of animated pictures, Sandy examines her painful joints and records if they are swollen. This is aided by a little camera provided by the cloud-based system that also measures the circumferences of her finger joints and toes. All these findings are automatically entered into Sandy's health databank where many data are already available, most notably those of her 'Healthbit' wearable device. The activity tracker has recorded a reduced number of steps: from 8500 down to an average to 4700 . She avoids stairs, but her cardiovascular system as constantly monitored by EKG, blood pressure and pulse rate is fine. There is also no fever.

Subsequently, Sandy goes to supermarket for shopping. However, prior to entering the store, there is a little booth that besides dry cleaning, shoe repairs and key cutting offers instant health services such as imaging diseased joints and blood-based biomarker analysis. Sandy enters this area, and a quick scan of the finger and wrist joints is performed using a novel laser-based scanning technique. Luckily, she is already equipped with a small implantable device underneath the skin of her forearm where special health data are stored, which need special data protection and she did not want to have in the cloud. Here, all her genomic data are stored thanks to a gift by her husband who donated her full genome sequencing as a gift for last Christmas. She puts her forearm in the reading device, and also some drops of blood are taken by a small lancet for further analysis in the machines in the basement of the supermarket run by robots.

These analyse the gene expression profile of the leucocyte subsets as well as the proteomic and immunomic profile. Macrophage activation is highly positive; there are no signs of infections, certain soluble factors indicative of an inflammatory joint disease are elevated, and $\operatorname{IgA}$ and $\operatorname{IgM}$ rheumatoid factors as well as ACPA are detected. There is also the expression of anti-Ro antibodies. The genomic profile is read out and reveals HLA shared epitope positivity as well as certain genes indicative of a reduced activity of enzymes involved in the metabolism of non-steroidal drugs.

So, which data are available to guide in Sandy's diagnosis? We have:

- Patient history.

- Joint status.

- Imaging data.

- Wearable device data.

- Genetic data.

- Gene expression data.

- Autoantibodies.

Based on the diagnostic algorithms, the computer calculates the diagnosis: rheumatoid arthritis with a probability of $>99 \%$. Who would disagree with this diagnosis? And interestingly, the patient has not been seen by a physician let alone a rheumatologist.

What will Sandy do now after she has received the diagnosis, of course with an empathic and comprehensive explanation by the computer, how well this disease can be treated? She asks the computer which therapeutic options are available, and it offers four treatment options to Sandy using detailed animated decision aids, carefully outlining the pros and cons of each therapy:

1. Biological A.

2. A tsDMARD.

3. Combination treatment with biological A plus a tsDMARD.

4. Biologic A, a tsDMARDs plus regulatory chimeric antigen receptor (CAR) T cells, a technology that had been developed in haemato-oncology and has now been introduced to treat autoimmune diseases.

Sandy opts for choice 4, since she feels that this combination-even though quite elaborate-offers the best chances of a cure. She is now finally referred to a 'Rheumatology 4.0 Unit' in a large university-based centre that will provide this treatment. Interestingly, Sandy's health insurance company requests to monitor her adherence to the quite expensive tsDMARD using digital monitoring with an ingestible event marker (IEM), a capsule that on entering the stomach sends a signal to a central remote tracking system via a patch and her smartphone. In addition, $\mathrm{T}$ cell cultures derived from her blood via leukapheresis are expanded to yield regulatory CAR T cells. A 3-monthly evaluation of the cellular gene expression profile is performed, and after 6 months, treatment is changed to therapy with CAR T cells due to a favourable gene expression profile. After 1 year, there is a complete drug-free remission without any further deterioration of imaging scans and no further treatment.

Is this case pure science fiction or will this scenario be possible in the future? Let's now do a reality check on what already is available now.

Many people believe that cloud-based voice service machines may replace our conventional means of entering data into a computer, pretty much as we already saw in the Star Trek movies many years ago. Classical keyboards and stand-alone computers will be nearly extinct in the future or be used only for dedicated tasks. Of course, a sophisticated program will be necessary to ask the right questions, analyse them and put them into context of the other data. Self-examination programs using pictures of diseased joints and skin are already available and used in certain early arthritis projects. ${ }^{1}$ Mobile devices will potentially extend the reach of specialists outside of the clinic setting. A forecast is that 6.3 billion smartphone subscriptions will exist by the year 
2021 and can therefore potentially provide inexpensive universal access to diagnostic care. ${ }^{2}$ There will also be a tremendous progress in wearable technology ${ }^{3}$ that already now records activity (steps, stairs and calories used) and cardiovascular data and will be extended to the constant monitoring of serum parameters such as glucose and potentially even $\mathrm{C}$ reactive protein. These will also help monitoring drug adherence using digital monitoring with an 'IEM'.

Implantable devices to store data are available as well ${ }^{5}$ and whole genome typing will be quite inexpensive in the future. Instant scanning methods to assess inflammation already document involved joints, currently using fluorescence optical imaging, ${ }^{6}$ which is invasive, but non-invasive methods will be developed. Already now (however by rather complicated methods) gene expression analysis is possible and can be compared with thousands of databases already publicly available. ${ }^{7}$ These can discriminate between states of viral, bacterial or other inflammations. Proteomics and autoantibody analysis can be done with sophisticated and rapid techniques. Thus, many of these data can already be recorded today, and the major step forward will then be the process, which is called 'cognitive computing' using elements of artificial intelligence as well as 'deep' and machine ('self') learning and which assesses structured (ie, laboratory, imaging and activity) and unstructured data (ie, text). Interestingly, machine learning had been developed to excel in games such as checkers and chess and was then transferred to several completely distinct disciplines. In medical terms, there is a very good explanation of machine learning by Obermeyer and Emanuel $^{8}$ :

\begin{abstract}
Machine learning... approaches problems as a doctor progressing through residency might: by learning rules from data. Starting with patient-level observations, algorithms sift through vast numbers of variables, looking for combinations that reliably predict outcomes. In one sense, this process is similar to that of traditional regression models: there are outcomes, covariates, and statistical functions linking the two. But where machine learning shines is in handling enormous numbers of predictors-sometimes, remarkably, more predictors than observations-and combining them in nonlinear and highly interactive ways. This capacity allows us to use new kinds of data, whose sheer volume or complexity would previously have made analyzing them unimaginable.
\end{abstract}

Recently, in dermatology, 'deep convolutional neural networks' were reported to achieve performance similar to all tested experts demonstrating an artificial intelligence capable of classifying skin cancer with a level of competence comparable to 'human' dermatologists using mobile devices. ${ }^{2}$

Going back to Sandy's treatment, we now have multitude of biologicals, tsDMARDs and also optimised strategies ${ }^{9}{ }^{10}$ how to use them wisely, especially in very early disease such is the case with Sandy. ${ }^{11}$ Of course, transferring the breath-taking CAR technology from haemato-oncology to autoimmune diseases may still be science fiction, but initial approaches are already made. $^{12}$

You may finally ask what the role of the physician may be in 10 years from now considering the scenario above. Certainly, many diagnostic algorithms will be performed by computer-aided systems, but the physician will still guide the patient through the various options, especially if it comes to difficult therapeutic decisions where a personal experience will be of great importance, potentially involving a multidisciplinary team. Of course, the actual treatment and management 'at the front line' will need doctors and nurses, particularly in emergency medicine and in a ward setting.

In conclusion, many of the approaches outlined above will use 'Big Data', which is based on the expectation that computerised algorithms performing calculation, data processing and automated reasoning tasks may extract new knowledge from otherwise unmanageable vast data sets. ${ }^{13}$ In rheumatology, high throughput technologies in molecular research already generated big data in rheumatology some 15 years ago. These included 'omics' technologies, such as genomics, transcriptomics and cytomics. In the future, functional analysis and interpretation will require adaptation of existing and the development of new software tools frequently based on machine learning. Structuring and evaluating the biological (medical) context will be essential and will not just be a mathematical problem. More and more extensive recording of disease characteristics and molecular processes in individual patients will generate personal big data and will require new strategies for management to develop datadriven individualised concepts resulting in a better diagnosis and treatment. Regarding our profession, this will also require new approaches in education and professional competence. Obermeyer and Lee have again very well addressed this, and thus I end with a quote ${ }^{14}$ :

There is little doubt that algorithms will transform the thinking underlying medicine. The only question is whether this transformation will be driven by forces from within or outside the field. If medicine wishes to stay in control of its own future, physicians will not only have to embrace algorithms, they will also have to excel at developing and evaluating them, bringing machine learning methods into the medical domain.

\section{Gerd R Burmester}

Department of Rheumatology and Clinical Immunology, Charité - University Medicine Berlin, Berlin, Germany

Correspondence to Dr Gerd R Burmester, Department of Rheumatology and Clinical Immunology, Charité - University Medicine Berlin, Berlin, 10117, Germany; gerd.burmester@charite.de

Competing interests None declared.

Patient consent Not required.

Provenance and peer review Commissioned; internally peer reviewed.

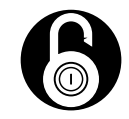

\section{OPEN ACCESS}

Open access This is an Open Access article distributed in accordance with the Creative Commons Attribution Non Commercial (CC BY-NC 4.0) license, which permits others to distribute, remix, adapt, build upon this work non-commercially, and license their derivative works on different terms, provided the original work is properly cited and the use is non-commercial. See: http://creativecommons.org/ licenses/by-nc/4.0/

(c) Article author(s) (or their employer(s) unless otherwise stated in the text of the article) 2018. All rights reserved. No commercial use is permitted unless otherwise expressly granted.

\section{Check for updates}

To cite Burmester GR. Ann Rheum Dis 2018;77:963-965.

Received 2 May 2018

Revised 7 May 2018

Accepted 7 May 2018

Published Online First 25 May 2018

\section{REFERENCES}

1 Schnelle Hilfe bei Rheuma. https://www.rheport.de/Start.aspx (accessed 02 May 2018).

2 Esteva A, Kuprel B, Novoa RA, et al. Dermatologist-level classification of skin cancer with deep neural networks. Nature 2017;542:115-8. 
3 Pevnick JM, Birkeland K, Zimmer R, et al. Wearable technology for cardiology: an update and framework for the future. Trends Cardiovasc Med 2018;28:144-50

4 Thasso. Abilify MyCite, the first digital pill. Where does all that silicone go? http:// thasso.com/abilify-mycite-the-first-digital-pill-where-does-all-that-silicon-go (accessed 02 May 2018).

5 New range of flexible implantable devices to launch later this month. https://www. xda-developers.com/140883-2 (accessed 02 May 2018).

6 Krohn M, Ohrndorf S, Werner SG, et al. Near-infrared fluorescence optical imaging in early rheumatoid arthritis: a comparison to magnetic resonance imaging and ultrasonography. J Rheumatol 2015;42:1112-8.

7 BioRetis. http://www.bioretis.com/ (accessed 02 May 2018)

8 Obermeyer Z, Emanuel EJ. Predicting the future - big data, machine learning, and clinical medicine. N Engl J Med 2016;375:1216-9.
9 Burmester GR, Pope JE. Novel treatment strategies in rheumatoid arthritis. Lancet 2017:389:2338-48.

10 Smolen JS, Breedveld FC, Burmester GR, et al. Treating rheumatoid arthritis to target: 2014 update of the recommendations of an international task force. Ann Rheum Dis 2016;75:3-15.

11 Bijlsma JWJ, Welsing PMJ, Woodworth TG, et al. Early rheumatoid arthritis treated with tocilizumab, methotrexate, or their combination (U-Act-Early): a multicentre, randomised, double-blind, double-dummy, strategy trial. Lancet 2016:388:343-55.

12 Biswas M, Kumar SRP, Terhorst C, et al. Gene therapy with regulatory T Cells: a beneficial alliance. Front Immunol 2018:9:554.

13 Bonin-Andresen M, Smiljanovic B, Stuhlmüller B, et al. [Relevance of big data for molecular diagnostics]. Z Rheumatol 2018;77:195-202.

14 Obermeyer Z, Lee TH. Lost in thought - the limits of the human mind and the future of medicine. N Eng/ J Med 2017;377:1209-11. 